\title{
Design of Coplanar Waveguide Matching Circuit for RF-CMOS Front-End
}

\author{
Haruichi Kanaya, Tetsuya Nakamura, Koji Kawakami, and Keiji Yoshida \\ Graduate School of Information Science and Electrical Engineering, Kyushu University, Fukuoka, 812-8581 Japan
}

\section{SUMMARY}

For enhanced performance with smaller transceiver size at microwave frequencies, a design method is proposed in which the impedance matching network is included in the filter and integrated with the antenna. In this paper, a low noise amplifier (LNA) and a power amplifier (PA) are designed by the CMOS process. A method is presented in which the input and output impedances are matched on the chip by a matching circuit using distributed transmission lines. As the distributed transmission lines, coplanar waveguides (CPW) are used for realization of miniaturized design. First, the RF characteristics of the CPW meander line are evaluated by an electromagnetic field simulator and are compared with measured results. Next, an amplifier integrated with matching circuits is designed and its performance is predicted by a simulator. The targeted frequency of operation is $2.4 \mathrm{GHz}$ for wireless LAN (IEEE802.11b). ( $) 2005$ Wiley Periodicals, Inc. Electron Comm Jpn Pt 2, 88(7): 19-26, 2005; Published online in Wiley InterScience (www.interscience.wiley.com). DOI 10.1002/ecjb.20161

\footnotetext{
Contract grant sponsors: Partly supported by VLSI Design and Education Center (VDEC), the University of Tokyo in collaboration with CADENCE and SYNOPSYS Corporation; and partly in collaboration with Innovation Plaza Fukuoka, Japan Science and Technology Corporation. It was partly supported by a Fukuoka project in the Cooperative Link of Unique Science and Technology for Economy Revitalization (CLUSTER) of the Ministry of Education, Culture, Sports, Science and Technology (MEXT); and partly supported by a Grant-in-Aid (14GS0218, 15760245) from MEXT and the Japan Society for the Promotion of Science.
}

Key words: RF-CMOS; wireless LAN; coplanar waveguide; impedance matching circuit.

\section{Introduction}

With the rapid development of the information society represented by mobile communications (IMT2000), wireless LAN, and satellite communications, the development of devices to realize communications systems with higher performance and higher efficiency is necessary. In order to meet this need, it is an urgent task to develop monolithic microwave integrated circuits (MMIC) in which filters, low-noise amplifiers (LNA), and power amplifiers (PA), as well as base band circuits and digital circuits, are integrated by applying CMOS circuits, with good digital circuit compatibility, to analog circuits $[1,2]$. Already in wireless LAN, a two-chip system has been developed for $\mathrm{RF}$ front ends for PC cards. However, the frequency selection filter and the harmonic rejection filter are externally installed.

Also, for $50-\Omega$ matching (conjugate matching) and noise matching with external circuits, spiral inductors are mounted on a CMOS chip in the case of low-noise amplifiers. But their size prevents miniaturized design. Further, since the spiral inductor has an extremely low $Q$ and may have self-resonance, it is difficult to operate at higher frequencies with high gain and low noise. Also, filter design is not possible.

As a design method eliminating the spiral inductor for future higher frequency operations, the authors have introduced a resonator configuration using a distributed

(C) 2005 Wiley Periodicals, Inc. 
(a)

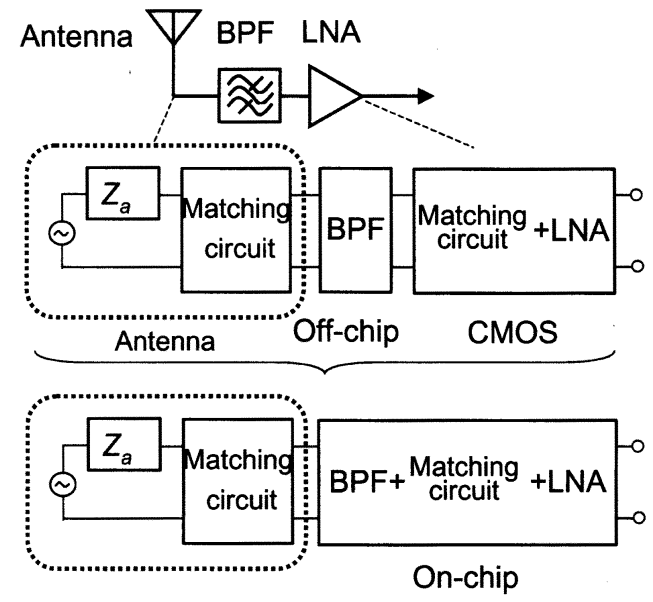

Fig. 1. Block diagram of the RF section.

(a) Conventional type. (b) On-chip type.

transmission line and an impedance inverter (inverter circuit). It is planned to realize on CMOS a circuit element combining a filter (bandpass design) and a matching circuit. The size of the distributed resonator can be reduced in inverse proportion to the frequency.

Since the coplanar waveguide (CPW) is used as a transmission line in which the signal line and the grounding conductors are on the same plane, coupling with CMOS active devices is easy and grounding is also simple. Hence, miniaturization of the devices is possible. Figure 1 shows a block diagram of the RF section. In the usual system, the filter is connected externally as shown in panel (a). The present authors have designed and published an antenna integrated with a filter in which the antenna, matching circuit, and filter are integrated together [3-5]. In the present research, a filter is designed on CMOS as shown in panel (b). The objective is to unify the input and output matching networks with the filter. The 2.4-GHz band used for wireless LAN (IEEE802.11b) is the targeted frequency.

\section{Design Method for Matching Circuits}

The present matching circuit is based on the theory of the Chebyshev bandpass filter (BPF) [6]. The BPF consists of distributed transmission lines and impedance inverters ( $K$ inverters, $K_{i, i+1}$ ). In usual wireless LAN cards, a chip dielectric filter is used as an external BPF. Its specifications are usually a passband of $100 \mathrm{MHz}$, an insertion loss of $1.5 \mathrm{~dB}, 0.5-\mathrm{dB}$ Chebyshev ripple, and an attenuation of $30 \mathrm{~dB}$ at $1.9 \mathrm{GHz}$. In order to meet these specifications, at least a three-stage BPF characteristic is needed. It is desirable to improve selectivity by increasing the number of stages in the BPF. Due to limited chip area, this paper uses a one-stage filter. The design formula for a one-stage BPF using the impedance inverters ( $K$ inverters) shown in Fig. 2 is

$$
\begin{gathered}
K_{0,1}=\sqrt{w} \sqrt{\frac{Z_{0} x_{1}}{g_{0} g_{1}}} \\
K_{1,2}=\sqrt{w} \sqrt{\frac{x_{1} Z_{0}}{g_{1} g_{2}}} \\
X_{1}=x_{1}\left(\frac{\omega}{\omega_{0}}-\frac{\omega_{0}}{\omega}\right)
\end{gathered}
$$

Here, $X_{1}$ is the reactance in the series resonator with a reactance slope parameter $x_{1}, w$ is the relative bandwidth, and $g_{i}$ is the filter parameter.

The equivalent circuit of the filter in Fig. 2 at the center part is shown in Fig. 3. Hence, the resistance ratio and the $Q$ value are given by

$$
\begin{aligned}
& \frac{R_{L}^{\prime}}{R_{S}^{\prime}}=\frac{g_{0}}{g_{2}} \\
& Q=\frac{x_{1}}{R_{S}^{\prime}+R_{L}^{\prime}}=\frac{g_{0} g_{1} g_{2}}{w\left(g_{0}+g_{2}\right)}
\end{aligned}
$$

Next, let us explain a miniaturization design method using a $\lambda / 4$ transmission line.

Figure 4 shows a new distributed impedance matching circuit proposed by the present authors using a $K$ inverter and a $\lambda / 4$ transmission line. Since the same design method can be used for both the input and output sides, only the input matching circuit is described. Here $Y_{L}$ is the input admittance of the LNA and is given by

$$
Y_{L}=\frac{1}{Z_{L}} \equiv G_{L}+j B_{L}
$$

In the compensation circuit, $\Delta l$ is determined by Eq. (7) in such a way that the susceptance $B_{L}$ of the LNA is canceled. Here, $C(\mathrm{~F} / \mathrm{m})$ is the capacitance per unit length of the distributed transmission line:

$$
\Delta l=-\frac{B_{L}}{\omega_{0} C}
$$

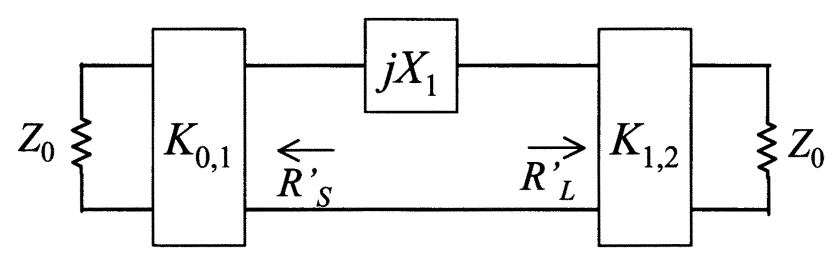

Fig. 2. Circuit model of the one-pole BPF with $K$ inverter. 


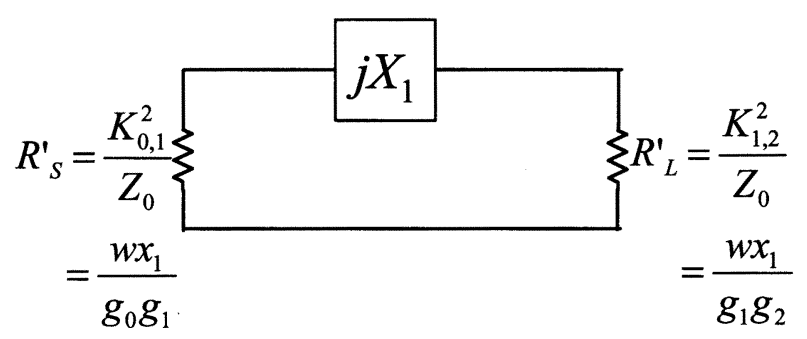

Fig. 3. Equivalent circuit model at center frequency.

Next, in Fig. $4, Z_{L}^{\prime}$ and $R_{S}^{\prime}$ can be written in terms of the characteristic impedance $Z_{1}$ and the electrical length $\theta$ of the $\lambda / 4$ transmission line as follows:

$$
\begin{aligned}
& Z_{L}^{\prime}=Z_{1}^{2} G_{L}+j X_{1} \equiv R_{L}^{\prime}+j X_{L}^{\prime} \\
& X_{1}=-Z_{1} \cot \theta \cong x_{1}\left(\frac{\omega}{\omega_{0}}-\frac{\omega_{0}}{\omega}\right) \\
& x_{1}^{\prime}=\frac{\pi}{4} Z_{1} \\
& R_{L}^{\prime}=Z_{1}^{2} G_{L} \\
& R_{S}^{\prime}=\frac{K_{0,1}^{2}}{Z_{0}}
\end{aligned}
$$

It is assumed in Eq. (8) that the input impedance of the LNA is sufficiently larger than $Z_{0}$ so that $\left|Y_{L}\right| \ll<Y_{0}$. Hence, the equivalent circuit at $\mathrm{A}-\mathrm{A}^{\prime}$ can be modified to that in Fig. 5.

To make this structure identical to the one-stage filter, it is found from resonant conditions (4) and (5) that

$$
\begin{aligned}
\frac{R_{L}^{\prime}}{R_{S}^{\prime}} & =\frac{Z_{0}}{K_{0,1}^{2}} Z_{1}^{2} G_{L}=\frac{g_{0}}{g_{2}} \\
Q & =\frac{x_{1}}{R_{S}^{\prime}+R_{L}^{\prime}}=\frac{Z_{0}}{K_{0,1}^{2}} \frac{x_{1}}{\left(1+\frac{g_{0}}{g_{2}}\right)} \\
& =\frac{g_{0} g_{1} g_{2}}{w\left(g_{0}+g_{2}\right)}
\end{aligned}
$$

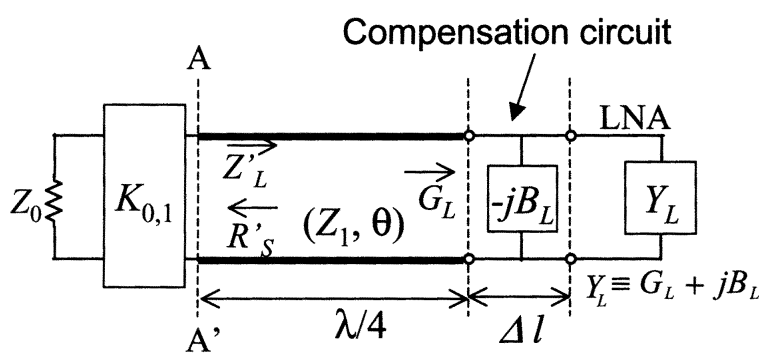

Fig. 4. Circuit model of the quarter-wavelength matching circuit.

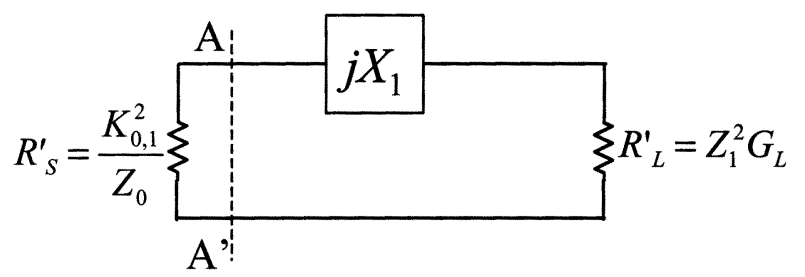

Fig. 5. Equivalent circuit model at $\mathrm{A}-\mathrm{A}^{\prime}$ in Fig. 4.

When these two equations are solved for $K_{0,1}$ and $Z_{1}$, the following are eventually obtained as design formulas:

$$
\begin{aligned}
& Z_{1}=\frac{\pi}{4} \frac{w}{g_{1} g_{2} G_{L}} \\
& K_{0,1}=\sqrt{w} \sqrt{\frac{Z_{0} x_{1}}{g_{0} g_{1}}} \\
& x_{1}=\frac{\pi}{4} Z_{1}
\end{aligned}
$$

Therefore, impedance matching is possible with the characteristic impedance $\left(Z_{1}\right)$ of the $\lambda / 4$ transmission line and $K_{0,1}$. Also, it is found from Eq. (16) that the desired bandwidth can be obtained by varying $w$. If noise matching is used, the above can also be applied by letting $Y_{L}=Y_{o p t}^{*}$ ( $Y_{\text {opt }}$ is the admittance minimizing the noise) [7].

\section{Design of Matching Circuit}

For microwave circuits, microstrip lines are often used. In order to design the matching circuit on CMOS, the present authors use coplanar waveguides (CPW). As shown in Fig. 6, CPW can realize grounding easily since the signal line and the grounding conductors are on the same plane. In addition, the characteristic impedance of the transmission line can be determined from the ratio of the signal line width and the ground plane separation. Hence, miniaturiza-

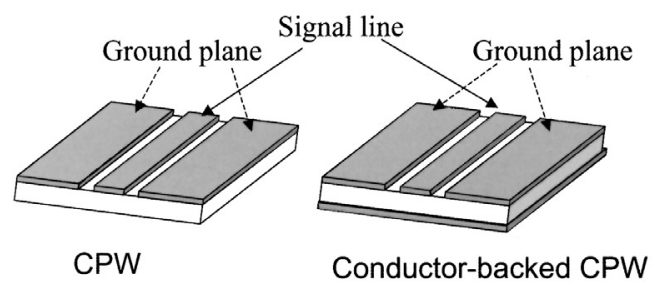

Fig. 6. Schematic diagram of the CPW structure. 


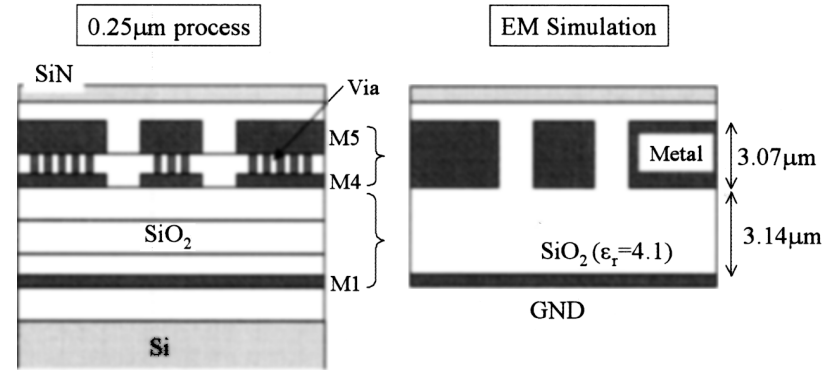

Fig. 7. Sectional views of the real process and EM-simulated condition.

tion is possible by proportional size reduction. Also, in comparison with microstrip lines, the electric field emissions are smaller, so that coupling to other circuits can be reduced. Further, in order to eliminate the influence of losses in the Si substrate, a conductor-backed CPW is used, combining the lowest level metallization (see Fig. 6). The Momentum simulator (Agilent Technology) is used for electromagnetic field simulation of the CPW.

Figure 7 shows a cross-sectional view of the substrate modeled in the electromagnetic field simulation. In order to reduce metal loss, metal layer 5 and metal layer 4 are connected with many vias. Note that the conductors are $\mathrm{Al}$ and the dielectric is $\mathrm{SiO}_{2}$. Simulations are performed using the physical constants for conductivity, permittivity, and dielectric dissipation. Figure 8 shows the layout image of the CPW matching circuit. As shown in Fig. 8, meander line configurations are used for the $\lambda / 4$ transmission line and the compensation circuit for miniaturization. A meander short stub is used as a $K$ inverter.

\section{Design of Matching Circuit}

Figure 9 shows a photograph of the measurement system for RF transmission characteristics. For measurement, air coplanar probes (Cascade Microtech) are used. As

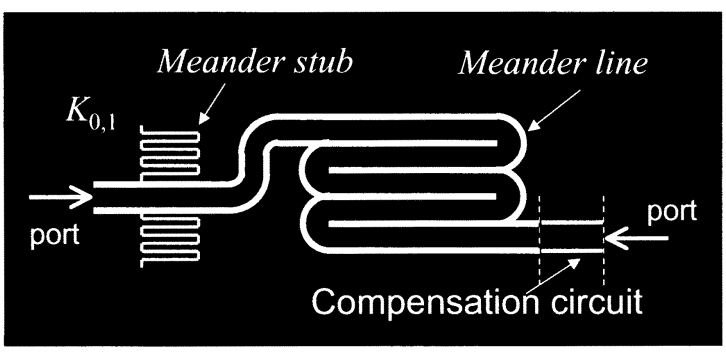

Fig. 8. Layout image of the CPW matching circuit.

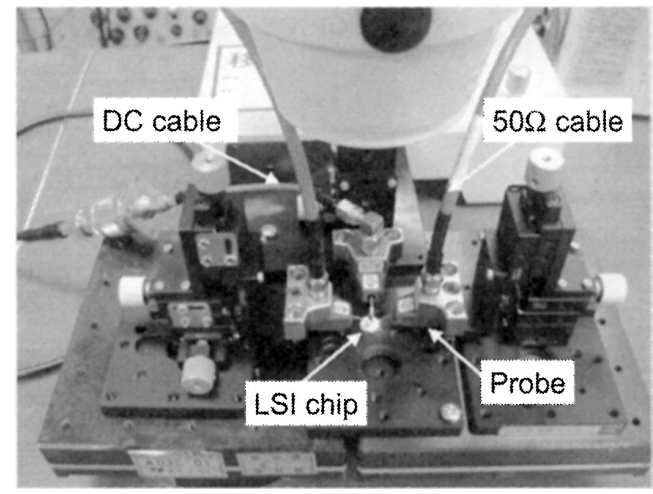

Fig. 9. Photograph of the RF measuring system.

a preliminary experiment, a test chip with a 50- $\Omega$ transmission line is evaluated prior to evaluation of the conductorbacked CPW. Figure 10 shows a chip photograph. The signal line width is $10 \mu \mathrm{m}$. The transmission line has a meander shape with a length of $13 \mathrm{~mm}$. The line length corresponds to a $\lambda / 4$ transmission line at $2.45 \mathrm{GHz}$. Figure 11 shows the measured results. For comparison, the simulation results obtained with the electromagnetic field simulator are also plotted. From the results of the electromagnetic field simulation, it is anticipated that attenuation of about $1 \mathrm{~dB}$ is possible near $2.45 \mathrm{GHz}$ due to loss of the aluminum electrode (electrode loss). On the other hand, in the experiment, an additional loss of $1 \mathrm{~dB}$ is seen in the experimental results. This is attributed to loss by the measurement PAD (including the connection with the coplanar probe). However, since the agreement with the results of the electromagnetic field simulator is reasonable, it is found that CPW design by the electromagnetic field simulator is possible. In the future, an increase of the operating frequency for improved communications speed is conceivable. Therefore, further miniaturization and loss reduction will be possible by means of reduced transmission line length.

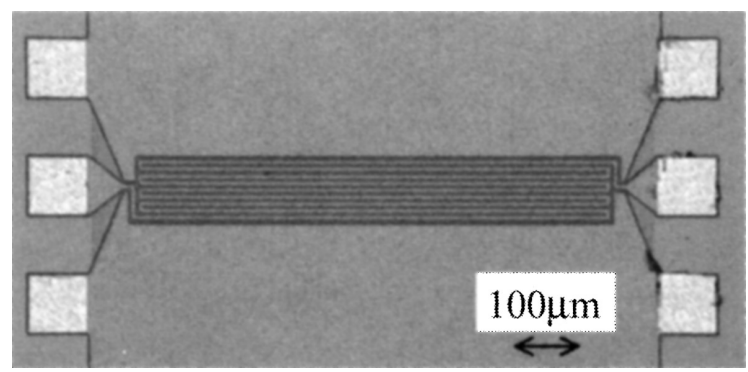

Fig. 10. Photograph of the conductor-backed CPW. 


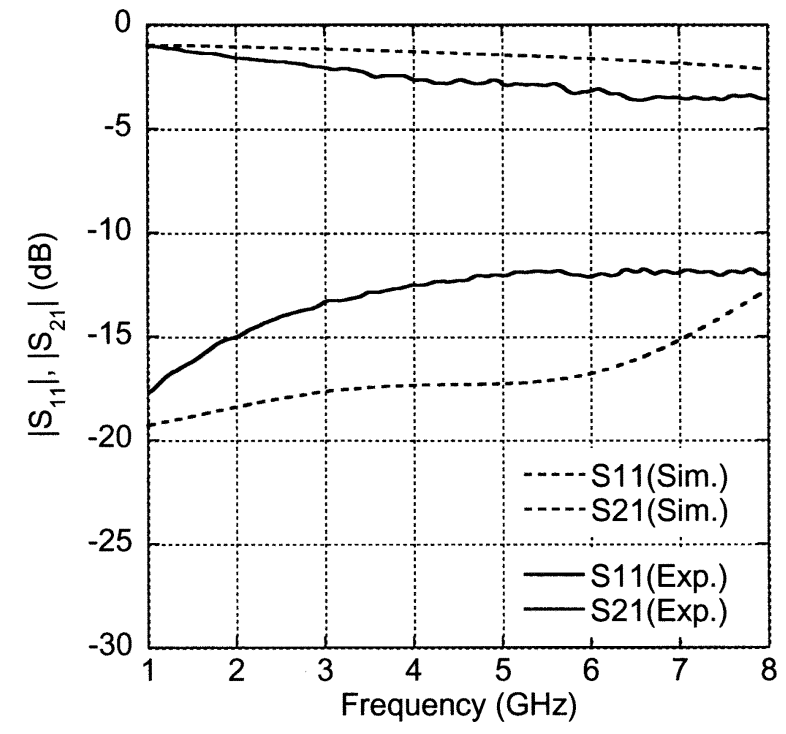

Fig. 11. Characteristics of the conductor-backed CPW.

\section{Design of LNA Integrated with a Matching Circuit}

Although design was carried out for both LNA and PA, only the design for the LNA is described here. In the design of the LNA, the $0.25-\mu \mathrm{m}$ CMOS process with TSMC is used. As the CAD tool, Virtuoso (Cadence) provided by VDEC is used. Also, for calculation of the input and output impedances and the noise figure of the LNA, ADS (by Agilent Technology) is used. Since an RF front end for the wireless LAN (for IEEE802.11b) is designed, a center frequency of $2.447 \mathrm{GHz}$ and a bandwidth of 100

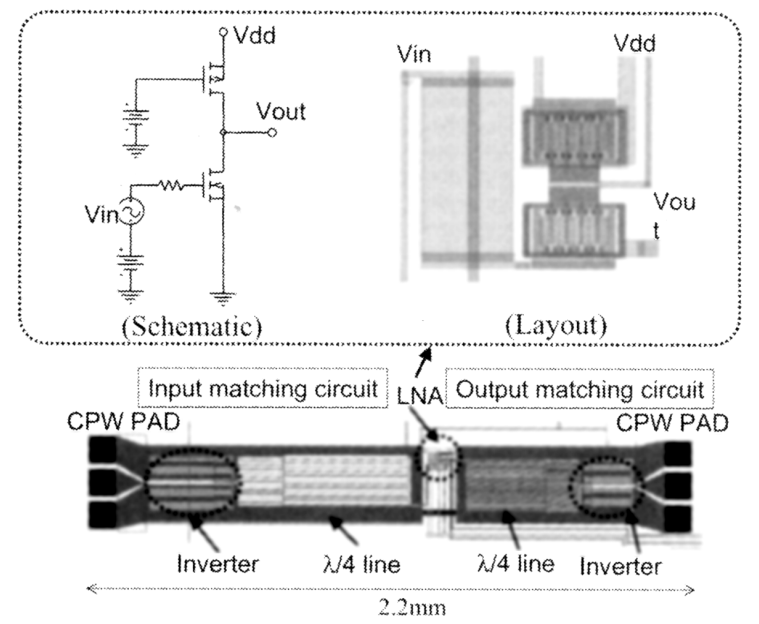

Fig. 12. Layout of input and output matching circuit connected with LNA.
$\mathrm{MHz}$ are chosen. The input and output matching circuits using the $K$ inverters and a $\lambda / 4$ transmission line are designed. The design theory is verified by means of electromagnetic field simulation.

First, the input and output impedances of the LNA at $2.45 \mathrm{GHz}$ are measured by the circuit simulator. Next, by using the obtained input and output impedance values, the matching circuit is designed by means of the electromagnetic field simulator. In the design, the port impedance of the electromagnetic field simulator is replaced by the input or output impedance of the LNA. The S parameters of the matching circuit section obtained by the electromagnetic field simulator are entered into the circuit simulator and the final results are obtained by combining them with the amplifier section. Figure 12 shows a chip layout of the LNA with an attached matching circuit integrated with a onestage filter as the input and output. For size reduction, a meander configuration with undulating transmission lines as shown in Fig. 10 is used. At the input and output sections, CPW pads are installed for measurement by the air coplanar probe. The area occupied by the amplifier section is $2.2 \times$ $0.2 \mathrm{~mm}$ including the input and output pads.

Figure 13 shows the frequency characteristics of the input power gain and the reflection coefficient. In the simulation results, the loss of the aluminum electrodes is taken into account but not the loss of the CPW obtained in the experiment. From the circuit simulation, the power gain is $15 \mathrm{~dB}$ at $2.45 \mathrm{GHz}$. From the reflection coefficient in Fig. 13 , matching around $2.45 \mathrm{GHz}$ is obvious. Also, as shown in Fig. 14, the noise figure of $1.8 \mathrm{~dB}$ is obtained at 2.45 GHz. In Fig. 15, the input and output impedances at the

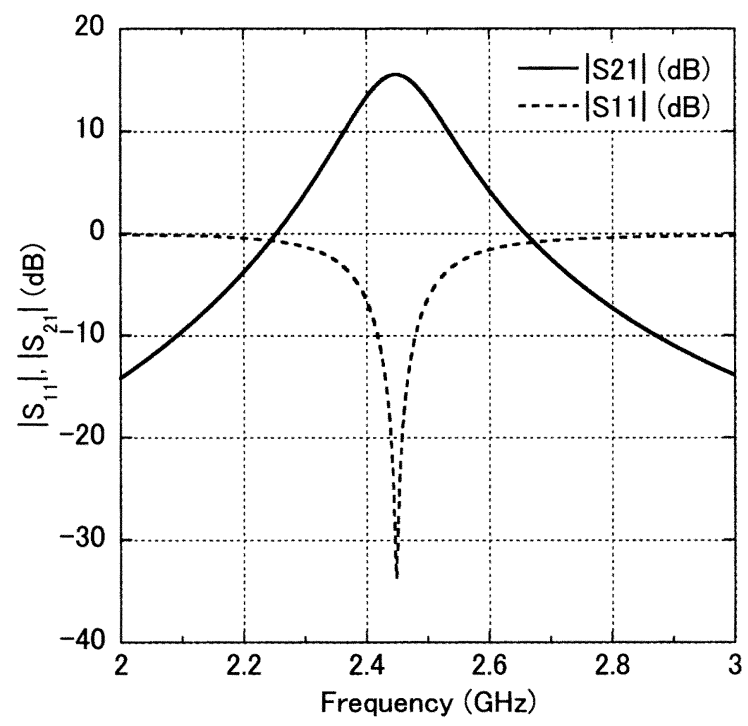

Fig. 13. Characteristics of the LNA with input and output matching circuit. 


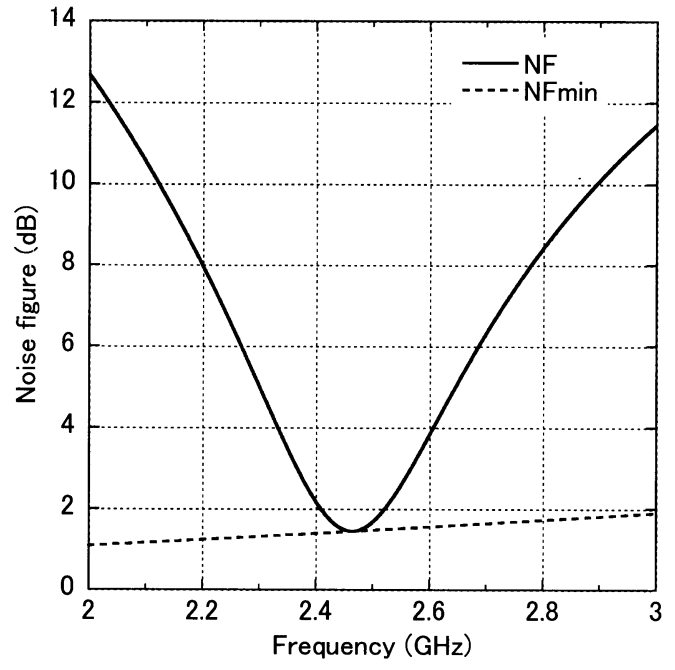

Fig. 14. Noise figure of the LNA with input and output matching circuit.

input and output sections are presented. Both input and output sections are almost matched to $50 \Omega$ in the real part and $0 \Omega$ in the imaginary part. By the present design method, a matching circuit using distributed transmission lines has become possible.

Usually, a small inductance is inserted between the source and the ground to make the real part of the impedance close to $50 \Omega$. However, the present design does not use a spiral inductor because elimination of the spiral inductor is extremely important for future enhancement of

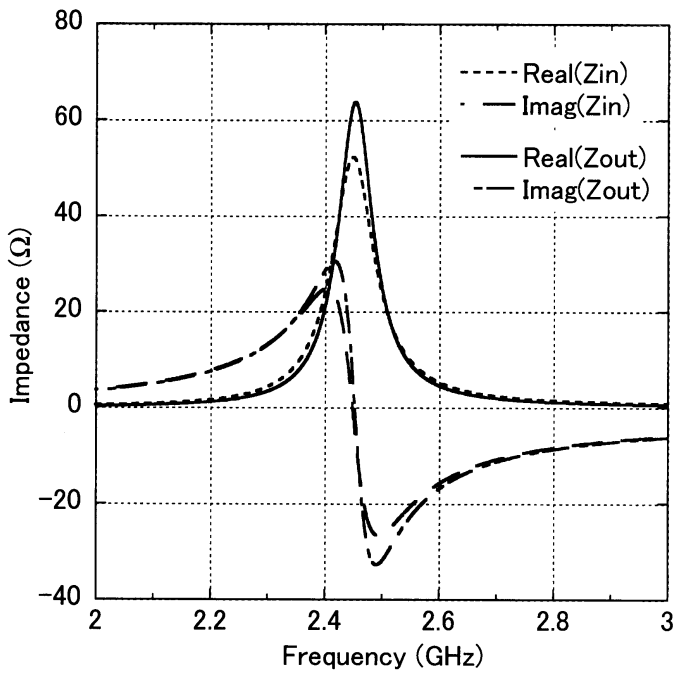

Fig. 15. Input and output impedance of the LNA with input and output matching circuit. the operating frequency and functionality, as explained in Section 1. As a result, the calculation of the matching condition from the input impedance of the LNA designed in this paper indicates that an inductance of about $60 \mathrm{nH}$ is needed at $2.45 \mathrm{GHz}$ if the conventional spiral inductor (lumped element) is used. On the other hand, if the CPW (distributed circuit) proposed here is used, the area ratio can be reduced to about $1 / 2$ and bandwidth design becomes possible. Hence, the chip size can be reduced dramatically. Further, if the spiral inductor is used in the present design rule, no other devices can be placed within a range of 50 $\mu \mathrm{m}$ due to magnetic field leakage from the inductor. On the other hand, the distributed transmission lines can be bent freely and are convenient for high degrees of integration.

\section{Conclusions}

By using a BPF formed with distributed transmission lines and inverter circuits, the filter and the matching circuit are integrated and realized on a CMOS chip. It is proposed in this way to design an RF-CMOS low noise amplifier and a power amplifier integrated with a filter. The analog circuit and the base band processor are placed on the same chip. The chip is presently under trial fabrication. The chip layout is shown in Fig. 16. In this figure, the matching circuit proposed in this paper is added only at the input of the LNA and the output of the PA. In general, lumped element devices such as spiral inductors cannot be used at higher frequencies due to self resonance. On the other hand, since the present matching circuit makes use of a resonant configuration, size reduction is possible as the frequency of operation is increased. Also, since filter circuit design is

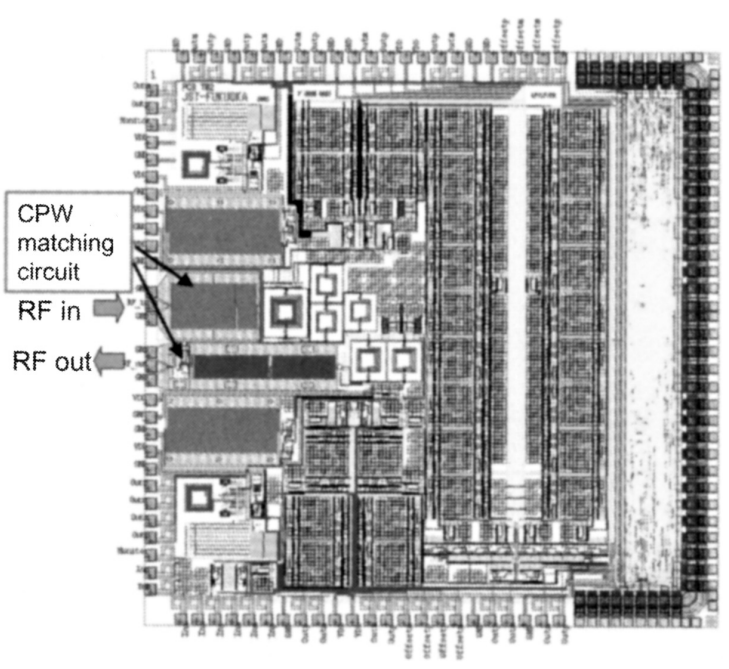

Fig. 16. Chip layout of RF-CMOS front-end (chip size: $5 \mathrm{~mm} \times 5 \mathrm{~mm}$ ). 
possible in the present matching circuit, it is possible to carry out integrated design with a BPF by using multistage matching circuits.

Acknowledgments. This work was partly supported by VLSI Design and Education Center (VDEC), the University of Tokyo in collaboration with CADENCE and SYNOPSYS Corporation; and partly in collaboration with Innovation Plaza Fukuoka, Japan Science and Technology Corporation. It was partly supported by a Fukuoka project in the Cooperative Link of Unique Science and Technology for Economy Revitalization (CLUSTER) of the Ministry of Education, Culture, Sports, Science and Technology (MEXT); and partly supported by a Grant-in-Aid (14GS0218, 15760245) from MEXT and the Japan Society for the Promotion of Science.

\section{REFERENCES}

1. Matsuzawa A. RF-SoC-expectations and required conditions. IEEE Trans Microwave Theory Tech 2002;50:245-253.
2. Ono M, Suematsu N, Kubo S, Nakajima K, Iyama Y, Takagi T, Ishida O. Si substrate resistivity design for on-chip matching circuit based on electro-magnetic simulation. IEICE Trans Electron 2001;E84-C:923929.

3. Yoshida K, Takahashi T, Kanaya H, Uchiyama T, Wang Z. Superconducting slot antenna with broadband impedance matching circuit. IEEE Trans Appl Supercond 2001;11:103-106.

4. Kanaya H, Koga Y, Fujiyama J, Urakawa G, Yoshida K. Design and performance of high Tc superconducting coplanar waveguide matching circuit for RFCMOS LNA. IEICE Trans Electron 2003;E86-C: 37-41.

5. Kanaya H, Koga Y, Urakawa G, Yoshida K. Design of HTS coplanar waveguide matching circuit for low noise CMOS-HTS receiver. IEEE Trans Appl Supercond 2003;13:1031-1034.

6. Matthaei G, Young L, Jones E. Microwave filters, impedance-matching networks, and coupling structures. McGraw-Hill; 1964. p 427-440.

7. Yoshida K, Kanaya H. Impedance matching circuit and its use for semiconductor devices and wireless communications equipment. Patent Application 2003-64128.

\section{AUTHORS (from left to right)}
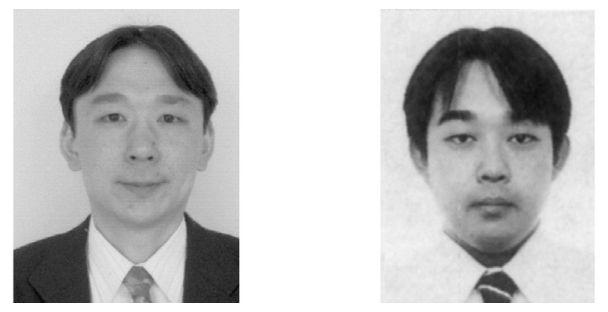

Haruichi Kanaya (member) graduated from the Department of Physics, Yamaguchi University, in 1990 and completed the accelerated doctoral program at Kyushu University in 1994. He was a special postdoctoral research associate supported by the Japan Society of Promotion for Science. He became a research associate at Yamaguchi University. After working as a doctoral research staff member at Massachusetts Institute of Technology, he became a research associate at the Graduate School of Information Science and Electrical Engineering, Kyushu University, in 1999. In 2003, he was appointed an associate professor with a joint appointment at the System LSI Research Center. He holds a D.Eng. degree. He has been engaged in research on the design and evaluation of system LSI for wireless communications. He received a 5th LSI IP Design Award for Development Promotion in 2003. He is a member of IEEE, the Applied Physics Society, the Japan Physical Society, and the Low Temperature Engineering Society.

Tetsuya Nakamura (student member) graduated from the Department of Electrical and Information Engineering, Kyushu University, in 2003 and is now a graduate student in the field of electronic devices at the Graduate School of Information Science and Electrical Engineering. He has been engaged in research on the design and evaluation of RF CMOS circuits. 
AUTHORS (continued) (from left to right)
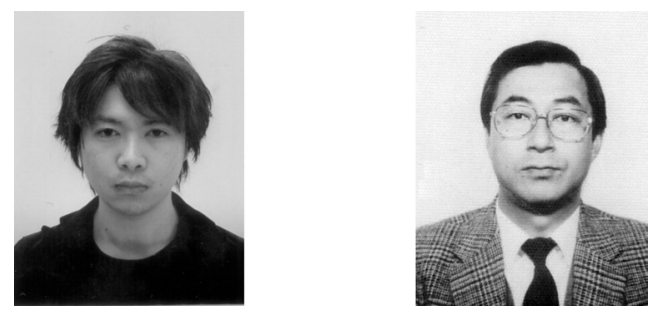

Koji Kawakami (student member) graduated from the Department of Electrical and Information Engineering, Kyushu University, in 2003 and is now a graduate student in the field of electronic devices at the Graduate School of Information Science and Electrical Engineering. He has been engaged in research on the design and evaluation of small planar filters.

Keiji Yoshida (member) graduated from the Department of Electronic Engineering, Kyushu University, in 1971 and completed the doctoral program in 1976. He is professor at the Graduate School of Information Science and Electrical Engineering. He holds a D.Eng. degree. He has been engaged in research on the design and development of high-speed optical communications devices and system LSI for wireless communications. He is a member of the Applied Physics Society and the Low Temperature Engineering Society. 